\title{
A polymorphism of the metabotropic glutamate receptor mGluR7 (GRM7) gene is associated with schizophrenia
}

Tsuyuka Ohtsuki ${ }^{1), 10)}$, Minori Koga ${ }^{1), 10)}$, Hiroki Ishiguro ${ }^{1), 10 *}$, Yasue Horiuchi ${ }^{1), 10)}$, Makoto Arai $^{2)}$, Kazuhiro Niizato ${ }^{3)}$, Masanari Itokawa ${ }^{2),}$, Toshiya Inada ${ }^{4)}$, Nakao Iwata ${ }^{5)}$, Shyuji Iritani $^{6)}$, Norio Ozaki ${ }^{6)}$, Hiroshi Kunugi ${ }^{7)}$,Hiroshi Ujike ${ }^{8)}$, Yuichiro Watanabe ${ }^{9)}$, Toshiuki Someya $^{9)}$, Tadao Arinami ${ }^{1), 10)}$

1) Department of Medical Genetics, Graduate School of Comprehensive Human Sciences, University of Tsukuba, Tsukuba, Ibaraki 305-8577, Japan.

2) Department of Schizophrenia Research, Tokyo Institute of Psychiatry, Tokyo 156-8585, Japan

3) Tokyo Metropolitan Matsuzawa Hospital, Department of Psychiatry, Tokyo 156-0057, Japan

4) Department of Psychiatry, Teikyo University School of Medicine, Chiba Medical Center, Anesaki 3426-3, Ichihara-shi, Chiba 299-0111 Japan

5) Department of Psychiatry, Fujita Health University School of Medicine, Toyoake, Aichi 470-1192, Japan

6) Department of Psychiatry, School of Medicine, Nagoya University, Nagoya 466-8550, Aichi, Japan

7) Department of Mental Disorder Research, National Institute of Neuroscience, National Center of Neurology and Psychiatry, 4-1-1 Ogawahigashi, Kodaira, Tokyo 187-8502, Japan

8) Department of Neuropsychiatry, Okayama University, Graduate School of Medicine, Dentistry \& Pharmaceutical Sciences, 2-5-1 Shikata-cho, Okayama 700-8558, Japan

9) Department of Psychiatry, Niigata University Graduate School of Medical and Denatal Sciences, Niigata 951-8510, Japan

10) CREST, Japan Science and Technology Agency, Kawaguchi-shi, Saitama 332-0012, Japan 
Running title: Association between GRM7 and schizophrenia

Key Words: glutamate, expression, luciferase, prefrontal

*Correspondence to

Hiroki Ishiguro, $\mathrm{MD}, \mathrm{PhD}$

Department of Medical Genetics, Graduate School of Comprehensive Human Sciences University of Tsukuba,Tsukuba, Ibaraki-ken, 305-8575, Japan

Tel: 81-298-53-3352, Fax: 81-298-53-3333

E-mail: hishigur@md.tsukuba.ac.jp

The present study was supported by Grant-in-Aid for Scientific Research on Priority Areas Research on Pathomechanisms of Brain Disorders (18023009) - from the Ministry of Education, Culture, Sports, Science and Technology of Japan.

Number of words in Abstract: 240

Number of words in text: 2501

Number of figures: 3

Number of tables: 2 


\begin{abstract}
Introduction: Glutamate dysfunction has been implicated in the pathophysiology of schizophrenia. The metabotropic glutamate receptors (mGluRs) are G-protein-coupled receptors. GRM7, the gene that encodes mGluR7, is expressed in many regions of the human central nervous system. The GRM7 gene is located on human chromosome 3p26, which has been suggested by linkage analysis to contain a susceptibility locus for schizophrenia.
\end{abstract}

Methods: We screened for mutations in all exons, exon/intron junctions, and promoter regions of the GRM7 gene in Japanese patients with schizophrenia and evaluated associations between the detected polymorphisms and schizophrenia. We examined the influence of one polymorphism associated with schizophrenia on the expression of GRM7 by dual luciferase assay in transfected cells.

Results: Twenty-five polymorphisms/mutations were detected in GRM7. Case-control analysis revealed a potential association of a synonymous polymorphism (371T/C, rs3749380) in exon 1 with schizophrenia in our case-control study of 2293 Japanese patients with schizophrenia and 2382 Japanese control subjects (allelic $p=0.009$ ). Dual luciferase assay revealed suppression of transcription activity by exon 1 containing this polymorphism and a statistically significant difference in the promoter activity between the $\mathrm{T}$ and $\mathrm{C}$ alleles. Conclusions: Our results support the possible association of a GRM7 gene polymorphism with genetic susceptibility to schizophrenia. 


\section{Introduction}

Schizophrenia is a severe psychiatric disorder, which is equally prevalent in men and women and affects approximately one percent of the population worldwide. Several neurotransmitter systems and functional networks within the brain have been found to be affected in patients with schizophrenia. The glutamatergic neuronal dysfunction hypothesis is one of the main explanatory hypotheses (Carlsson et al., 1997). Glutamate is the primary excitatory neurotransmitter in brain. It is contained as a neurotransmitter in approximately $60 \%$ of brain neurons, including almost all cortical pyramidal neurons. Further, virtually $100 \%$ of brain neurons contain some type of glutamate receptor. Glutamate mediates its effects on the central nervous system via both ionotropic and metabotropic receptors. The metabotropic glutamate receptors (mGluRs), which are G protein-coupled receptors, are divided into 3 groups on the basis of sequence homology, putative signal transduction mechanisms, and pharmacologic properties (Nakanishi 1994; Pin and Duvoisin 1995). The mGluRs in group I are mGluR1 and mGluR5, those in group II are mGluR2 and mGluR3, and those in group III are mGluRs 4, 6, 7, and 8. Group II and group III mGluRs are linked to inhibition of the cyclic AMP cascade but differ in their agonist selectivities.

mGluR7 is the most highly conserved mGluR subtype across mammalian species (Flor et al., 1997). Makoff et al (1996) observed by in situ hybridization that GRM7 is expressed in many areas of the human brain, especially the cerebral cortex, hippocampus, and cerebellum. mGluR7 is localized directly in the presynaptic zone of the synaptic cleft of glutamatergic synapses (Kinoshita et al 1998; Kosinski et al 1999), where it is thought to act as an autoreceptor that is activated by glutamate released from the presynaptic terminal during action potentials. Furthermore, mGluR7 is thought to be a key player in shaping synaptic responses at glutamatergic synapses as well as in regulating key aspects of inhibitory GABAergic transmission (Kinoshita et al 1998; Kosinski et al 1999).

mGluR7 has putative roles in anxiety, emotional responses, and spatial working memory (Callaerts-Vegh et al 2006; Cryan et al 2003; Mitsukawa et al 2006). Cognitive 
dysfunction is estimated to occur in $75 \%-85 \%$ of patients with schizophrenia, often precedes the onset of other symptoms (Reichenberg et al 2006). Working memory is one of primary cognitive domains that are crucial for developing targets for the treatment of cognition in schizophrenia (Nuechterlein et al 2004).

mGluR7 ablation causes dysregulation of the hypothalamic-pituitary-adrenal axis and increases hippocampal BDNF protein levels (Mitsukawa et al 2005). Dysregulation of BDNF production or release is associated with neuropsychiatric disorders, such as schizophrenia (Kelley 2004; Harrison and Weinberger 2005). Association between the val66met polymorphism of the BDNF gene and hippocampal volume in human, particularly in patients with schizophrenia (Szeszko PR et al 2005).

In the present study, we examined the GRM7 gene as a candidate for schizophrenia.

\section{Materials and Methods}

Subjects

All subjects were of Japanese descent and were recruited from the main island of Japan. Patients with schizophrenia were diagnosed according to the Diagnostic and Statistical Manual of Mental Disorders, Fourth Edition (DSM-IV). Control subjects were mentally healthy, unrelated subjects with no self-reported family history of mental illness within second-degree relatives. We sequenced the 5' region, exons and exon-intron boundaries of the GRM7 gene in 32 patients (mutation screening patients) with schizophrenia (mean age $\pm \mathrm{SD}$, $46.5 \pm 10.9$ years; 17 men and 15 women) to identify polymorphisms. We then genotyped 576 patients including the mutation screening patients (mean age \pm SD, $46.6 \pm 14.8$ years; 322 men and 254 women) and 576 control subjects (mean age \pm SD, $46.8 \pm 12.9$ years; 268 men and 308 women) ( $1^{\text {st }}$ association population) with Predesigned TaqMan single nucleotide polymorphism (SNP) genotyping assays. When Predesigned TaqMan SNP genotyping assays were not available, we performed direct sequencing of DNAs from 96 patients including the mutation screening patients (mean age \pm SD, $50.3 \pm 13.1$ years; 55 men and 40 women) and 
96 control subjects (mean age \pm SD, $53.6 \pm 9.1$ years; 42 men and 54 women). For SNPs for which an association with schizophrenia was suggested in the first association population, we performed genotyping in an independent sample of 1817 patients (mean age \pm SD, $45.5 \pm$ 14.1 years; 962 men and 855 women) and 1728 control subjects (mean age \pm SD, $46.2 \pm 13.6$ years; 958 men and 770 women) (confirmation population). The present study was approved by the ethics committees of the University of Tsukuba and participating institutes. All participants provided written informed consent.

\section{DNA isolation and genotyping}

DNAs were extracted from peripheral lymphocytes by standard phenol-chloroform extraction. The genomic structure of GRM7 was determined from the University of California at Santa Cruz (UCSC) database (http://genome.ucsc.edu/cgi-bin/hgGateway) and the National Center for Biotechnology Information (NCBI) database (http://www.ncbi.nlm.nih.gov/). To screen for polymorphisms, we performed direct sequencing of genomic DNAs with a Big Dye Terminator Cycle Sequencing Kit and the ABI PRISM 3100 Genetic Analyzer (Applied Biosystems, Foster City, CA, USA). All exons, exon-intron junctions, and $1.6 \mathrm{~kb}$ of the 5' flanking region of GRM7 were amplified from the genomic DNAs of 24 randomly selected patients. The sequences of primers and conditions used for amplification for the mutation screening are available upon request. We genotyped polymorphisms with the TaqMan SNP Genotyping Assay (Applied Biosystems) and ABI PRISM 7900HT Sequence Detection System (Applied Biosystems).

Real-time quantitative reverse transcription-polymerase chain reaction (RT-PCR)

Total RNA was isolated from cultured cells with the SV Total RNA Isolation System (Promega, Madison, WI, USA). cDNA was synthesized from RNA with Revertra Ace (Toyobo, Tokyo, Japan) and oligo dT primer. Expression of GRM7 was quantified by real-time quantitative RT-PCR with the TaqMan Gene Expression Assay and ABI PRISM 
7900HT Sequence Detection System (Applied Biosystems) per the manufacturer’s instructions. Primers and probes were purchased from Applied Biosystems (Assays-on-Demand Assay ID: Hs00179051_m1). GAPDH was used as an internal control. Data were collected and analyzed with Sequence Detector Software (SDS) version 2.1 (Applied Biosystems) and the standard curve method.

\section{Luciferase reporter assay}

To assay promoter activity of the 5'-flanking region and exon 1 of the GRM7 gene, 9 fragments of the 5' region were cloned into the pGL3-Basic plasmid with and without a Simian virus 40 enhancer sequence (Promega, Madison, WI, USA). The day before transfection, NH-12 cells (Japanese Collection of Research Bioresources Gene Bank, http://genebank.nibio.go.jp/gbank/index_e.html) were plated at 1 X $10^{5}$ cells per well in a 24-well plate and grown in DMEM (Sigma, St. Louis, MO, USA) supplemented with $10 \%$ fetal bovine serum (FBS) (Equitech-Bio, Kerrville, TX, USA). One microgram of each test plasmid was transiently cotransfected into the cells with $0.1 \mu \mathrm{g}$ of pRL-TK plasmid (an internal standard reporter) (Promega) with Lipofectamine 2000 (Invitrogen) per manufacturer's protocol. After $48 \mathrm{~h}$, the dual-luciferase assay was performed with a PicaGene Dual SeaPansy Kit (Toyo Ink, Tokyo, Japan) according to the manufacturer's instructions.

\section{Statistics}

Deviation from predicted Hardy-Weinberg frequencies was examined by chi-square test. Individual allelic associations were examined by Fisher's exact test. Genotypic associations were examined by Armitage's Trend Test for the reasons discussed by Devlin and Roeder (Devlin and Roeder 1999). A significant association was defined when the given $p$ value for allelic or genotypic tests was less than $5 \%$ (uncorrected $p<0.05$ ) and the same association was confirmed in an independent population with $p<0.05$. Linkage 
disequilibrium (LD) between polymorphisms and haplotype block structures was evaluated with Haploview software version 3.11 (Barrett et al 2005). Haplotype blocks were generated with the default algorithm taken from Gabriel et al (Gabriel et al 2002). Haplotypic associations with disorders were examined with Haploview software, which performs association tests on the set of blocks selected by obtaining counts for case control association tests by summing the fractional likelihoods of each individual for each haplotype by the EM algorithm.

\section{Results}

Twenty-five polymorphisms/mutations were detected in the exons, exon-intron junctions, and 5'-flanking region of the GRM7 gene (Figure 1). Genotyping was carried out for all detected polymorphisms except rare variants with allele frequencies $<0.05$ and polymorphisms in LD with each other $\left(r^{2}=1\right)$. Among these SNPs, 1724A/G (rs34373930), 1938C/T (rs7614915), IVS8+49T/A (T/A at the position of 49 base-pair starting from the $\mathrm{G}$ of the donor site of intron 8), and 2345C/T (rs1485175) were in complete LD ( $\left.r^{2}=1\right)$, and IVS8+114T/C and 2384G/A (rs1485174) were also in complete LD $\left(r^{2}=1\right)$ in 24 screening samples. Therefore, we genotyped the 2345C/T and 2384G/A polymorphisms as representative SNPs. The IVS9+97C/T, 2912T/C, and 3292(A)3-4 (rs3840229) SNPs were genotyped in 96 patients and 96 controls by direct sequencing, and the other 12 SNPs were genotyped in 576 patients and 575 control subjects by TaqMan SNP Genotyping Assay (Applied Biosystems). The genotypic distributions of these 15 SNPs did not deviate significantly from Hardy-Weinberg equilibrium $(p>0.05)$. A synonymous polymorphism (371T/C) in exon 1 of GRM7 showed a potential association for schizophrenia (allelic uncorrected $p=0.04$ ) (Table 1). We did not detect a significant association with schizophrenia of any of the other 14 SNPs, including the Tyr433Phe polymorphism (allelic uncorrected $p=$ 0.33 ; genotypic uncorrected $p=0.63$ ), which was previously reported to be associated 
(Bolonna et al 2001). The haplotype blocks consisted of IVS1-76T/C, IVS1-67A/G, and IVS1-23G/A and of 2345C/T and 2384A/G. No significant haplotypic association was suggested for these 2 haplotype blocks ( $p>0.05$ ). We confirmed the association of the 371T/C polymorphism with schizophrenia in an independent population of 1717 patients and 1807 control subjects and confirmed the association (allelic $p=0.03$, one-sided) (Table 2). The allelic $p$ value of the association in the total population of 2293 patients with schizophrenia and 2382 control subjects was 0.009 (Table 2). According to the HapMap database (http://www.hapmap.org/index.html), the 371T/C polymorphism (rs3749380) was not in the LD block and was not in LD with other SNPs within $80 \mathrm{~kb}\left(r^{2}<0.7\right)$. Weak LD was observed with rs458351 ( $D^{\prime}=0.89$ and $\left.r^{2}=0.69\right)$, which is $30 \mathrm{~kb}$ upstream of rs3749380.

Expression of GRM7 mRNA was assessed by RT-PCR in 7 different human cell lines (IMR-32, NH-12, TN-2, NB-1, SCCH-26, A172, and T98G). GRM7 was expressed in NH-12 and SCCH-26 cells (data not shown). Because expression in NH-12 cells was higher than in SCCH-26 cells, we used NH-12 cells, a human cell line derived from neuroblastoma, for luciferase assays. Dual luciferase assay revealed that the strongest promoter activity for GRM7 was contained in the 1-kb fragment upstream of the ATG site of exon 1. However, a promoter construct containing the sequence from the ATG to the end of exon 1 showed significantly lower activity, indicating that the 371T/C polymorphism is in a regulatory region. When an SV40 enhancer was added downstream of the luciferase gene, the 371C allele showed significantly higher promoter activity than the $371 \mathrm{~T}$ allele (Figure 2).

\section{Discussion}

In the present study, we examined associations between polymorphisms in the GRM7 gene and schizophrenia. Weak association was found for a synonymous SNP (371T/C) in exon 1 in the first association population, and this association was replicated in the confirmation population. The T allele, which is associated with schizophrenia, has lower promoter activity than the $\mathrm{C}$ allele. On the basis of this finding, we hypothesized that lower 
expression of mGluR7 may increase risk of developing schizophrenia, though studies of the expression of GRM7 in brains of patients with schizophrenia have not been reported.

mGluR7 was the first group III mGluR found to be enriched presynaptically at active zones of hippocampal pyramidal cells (Shigemoto et al 1996). The low affinity of mGluR7 for glutamate suggests that mGluR7 might act as a "low-pass filter" that suppresses release of glutamate only when action potentials arriving at a high frequency produce massive glutamate release. The interaction with PICK1 (protein interacting with PRKCA 1) is crucial for the clustering of mGluR7 at presynaptic release sites (Boudin et al 2000). PICK1 is reported to be associated with schizophrenia (Dev and Henley 2006; Fujii et al 2006).

In the present study, we found an association between a functional SNP, 371T/C, in the gene encoding mGluR7 and schizophrenia. Recently, a genome-wide association study of 2000 individuals with bipolar disorder and 3000 control subjects found a strong association of SNP marker rs1485171 ( $p=9.7 \times 10^{-5}$ ) in GRM7 with bipolar disorder (Consortium 2007; Consortium. 2007). Therefore, genetic variations in the GRM7 gene may be involved in both schizophrenia and bipolar disorder.

An association between Tyr433Phe polymorphism of GRM7 and schizophrenia was reported (Bolonna et al 2001). However, we failed to detect the association (allele, $p=0.33$; genotype, $p=0.63)$. Recently, a copy number variation of the GRM7 locus has been reported in patients with schizophrenia (Wilson et al 2006). In the present study, we did not observe significant deviation from Hardy-Weinberg expectancy of the genotypic distributions of SNPs, indicating that copy number variations at the SNP examined in the present study are not common and are unlikely.

In the present study, the association of 371T/C with schizophrenia was observed in two independent case control populations. However, its weak association (OR = 1.12) requires replication studies in large sample populations of more than 2000 cases and 2000 control subjects with a power greater than 0.8 . We believe that GRM7 is an interesting target worth such studies for schizophrenia and other psychiatric disorders. 


\section{References}

Barrett JC., Fry B., Maller J., Daly MJ., 2005. Haploview: analysis and visualization of LD and haplotype maps. Bioinformatics 21 (2) 263-265

Bolonna AA., Kerwin RW., Munro J., Arranz MJ., Makoff AJ., 2001. Polymorphisms in the genes for mGluR types 7 and 8: association studies with schizophrenia. Schizophr Res. 47 (1) 99-103.

Boudin H., Doan A., Xia J., Shigemoto R., Huganir RL., Worley P., Craig AM., 2000. Presynaptic clustering of mGluR7a requires the PICK1 PDZ domain binding site. Neuron 28 (2) 485-497.

Callaerts-Vegh Z., Beckers T., Ball SM., Baeyens F., Callaerts PF., Cryan JF., Molnar E., D'Hooge R., 2006. Concomitant deficits in working memory and fear extinction are functionally dissociated from reduced anxiety in metabotropic glutamate receptor 7-deficient mice. J Neurosci. 26 (24) 6573-6582.

Carlsson A., Hansson LO., Waters N., Carlsson ML., 1997. Neurotransmitter aberrations in schizophrenia: new perspectives and therapeutic implications. Life Sci. 61 (2) 75-94.

Consortium WTCC, 2007. Genome-wide association study of 14,000 cases of seven common diseases and 3,000 shared controls. Nature 447 (7145) 661-678.

Cryan JF., Kelly PH., Neijt HC., Sansig G., Flor PJ., van Der Putten H., 2003. Antidepressant and anxiolytic-like effects in mice lacking the group III metabotropic glutamate receptor mGluR7. Eur J Neurosci. 17 (11) 2409-2417.

Dev KK., Henley JM., 2006. The schizophrenic faces of PICK1. Trends Pharmacol Sci. 27 (11) 574-579.

Devlin B., Roeder K., 1999. Genomic control for association studies. Biometrics 55 (4) 997-1004.

Fujii K., Maeda K., Hikida T., Mustafa AK., Balkissoon R., Xia J., Yamada T., Ozeki Y., Kawahara R., Okawa M., Huganir RL., Ujike H., Snyder SH., Sawa A., 2006. Serine racemase binds to PICK1: potential relevance to schizophrenia. Mol Psychiatry 11 (2) 150-157.

Gabriel SB., Schaffner SF., Nguyen H., Moore JM., Roy J., Blumenstiel B., Higgins J., DeFelice M., Lochner A., Faggart M., Liu-Cordero SN., Rotimi C., Adeyemo A., Cooper R., Ward R., Lander ES., Daly MJ., Altshuler D., 2002. The structure of haplotype blocks in the human genome. Science 296 (5576) 2225-2229.

Harrison PJ., Weinberger DR., 2005. Schizophrenia genes, gene expression, and neuropathology: on the matter of their convergence. Mol Psychiatry 10 (1) 40-68.

Kinoshita A., Shigemoto R., Ohishi H., van der Putten H., Mizuno N., 1998. Immunohistochemical localization of metabotropic glutamate receptors, mGluR7a and mGluR7b, in the central nervous system of the adult rat and mouse: a light and electron microscopic study. J Comp Neurol. 393 (3) 332-352.

Kosinski CM., Risso Bradley S., Conn PJ., Levey AI., Landwehrmeyer GB., Penney JB, Jr., Young AB., Standaert DG., 1999. Localization of metabotropic glutamate receptor 7 mRNA and mGluR7a protein in the rat basal ganglia. J Comp Neurol. 415 (2) 266-284.

Mitsukawa K., Mombereau C., Lotscher E., Uzunov DP., van der Putten H., Flor PJ., Cryan JF., 2006. Metabotropic glutamate receptor subtype 7 ablation causes dysregulation of the HPA axis and increases hippocampal BDNF protein levels: implications for stress-related psychiatric disorders. Neuropsychopharmacology 31 (6) 1112-1122.

Mitsukawa K., Yamamoto R., Ofner S., Nozulak J., Pescott O., Lukic S., Stoehr N., Mombereau C., Kuhn R., McAllister KH., van der Putten H., Cryan JF., Flor PJ., 2005. A selective metabotropic glutamate receptor 7 agonist: activation of receptor signaling via an allosteric site modulates stress parameters in vivo. Proc Natl Acad Sci U S A 102 (51) 18712-18717.

Nakanishi S., 1994. Metabotropic glutamate receptors: synaptic transmission, modulation, and plasticity. Neuron 13 (5) 1031-1037. 
Nuechterlein KH., Barch DM., Gold JM., Goldberg TE., Green MF., Heaton RK., 2004. Identification of separable cognitive factors in schizophrenia. Schizophr Res. 72 (1) 29-39.

Pin JP., Duvoisin R., 1995. The metabotropic glutamate receptors: structure and functions. Neuropharmacology 34 (1) 1-26.

Reichenberg A., Weiser M., Caspi A., Knobler HY., Lubin G., Harvey PD., Rabinowitz J., Davidson M., 2006. Premorbid intellectual functioning and risk of schizophrenia and spectrum disorders. J Clin Exp Neuropsychol. 28 (2) 193-207.

Shigemoto R., Kulik A., Roberts JD., Ohishi H., Nusser Z., Kaneko T., Somogyi P., 1996. Target-cell-specific concentration of a metabotropic glutamate receptor in the presynaptic active zone. Nature 381 (6582) 523-525.

Szeszko PR., Lipsky R., Mentschel C., Robinson D., Gunduz-Bruce H., Sevy S., Ashtari M., Napolitano B., Bilder RM., Kane JM., Goldman D., Malhotra AK., 2005.

Brain-derived neurotrophic factor val66met polymorphism and volume of the hippocampal formation. Mol Psychiatry 10 (7) 631-636.

Wilson GM., Flibotte S., Chopra V., Melnyk BL., Honer WG., Holt RA., 2006. DNA copy-number analysis in bipolar disorder and schizophrenia reveals aberrations in genes involved in glutamate signaling. Hum Mol Genet. 15 (5) 743-749. 


\section{FIGURE LEGENDS}

Figure 1. Schematic representation of the GRM7 gene and relevant mutations/polymorphisms (A), linkage disequilibrium plot of the Japanese population from HapMap database (B), and between SNPs genotyped in this study shown in Table 1 (C). Figures in rhombus are D’ between SNPs and filled rhombus without figures indicates D' = 1 in (C).

Figure 2. Luciferase assays.

(A) Schematic representation of the GRM7 gene and reporter gene constructs.

(B) Transcriptional activity of various constructs of the 5' region and exon 1 of the human GRM7 gene in NH-12 cells. The region indicated by fine lines were not included in the constructs. Cotransfections were performed with pRL-TK (Renilla luciferase) to normalize transfection efficiency. Luciferase activity was assayed $48 \mathrm{~h}$ after transfection. Relative ratio of expression is shown as relative to that of pGL3-Basic, which was set at 1. The mean \pm SEM was calculated from triplicate assays.

(C) Effect of the 371C/T polymorphism on GRM7 promoter activity. This assay was performed with pGL3-enhancer vector, because the constructs that contain GRM7 exon 1 show extremely low luciferase activity. 
Table 1. Genotypic and allelic distributions of polymorphisms in the GRM7 gene

\begin{tabular}{|c|c|c|c|c|c|c|c|c|c|c|c|c|c|c|}
\hline \multirow{4}{*}{$\begin{array}{l}\text { Polymorphism } \\
\text { 371T/C (rs3749 }\end{array}$} & \multirow{3}{*}{$\begin{array}{l}\text { Population } \\
\text { 0) } \\
\text { Patients }\end{array}$} & \multirow[t]{2}{*}{$\mathrm{n}$} & \multicolumn{6}{|c|}{ Genotype count (frequency) } & \multirow[t]{2}{*}{$p$} & \multicolumn{4}{|c|}{ Allele count (frequency) } & \multirow[t]{2}{*}{$p$} \\
\hline & & & \multicolumn{2}{|c|}{ CC } & \multicolumn{2}{|c|}{$\mathrm{TC}$} & \multicolumn{2}{|c|}{ TT } & & \multicolumn{2}{|c|}{$\mathrm{C}$} & \multicolumn{2}{|r|}{$\mathrm{T}$} & \\
\hline & & 576 & 241 & $(0.42)$ & 256 & $(0.44)$ & & $(0.14)$ & & 738 & $(0.64)$ & 414 & $(0.36)$ & \\
\hline & Controls & 575 & 274 & $(0.48)$ & 236 & $(0.41)$ & 65 & $(0.11)$ & 0.04 & 784 & $(0.68)$ & 366 & $(0.32)$ & 0.04 \\
\hline \multicolumn{2}{|c|}{ IVS1-76T/C (rs3749450) } & & \multicolumn{2}{|c|}{$\mathrm{CC}$} & \multicolumn{2}{|c|}{ TC } & \multicolumn{2}{|c|}{ TT } & & \multicolumn{2}{|c|}{ C } & \multicolumn{2}{|r|}{$\mathrm{T}$} & \\
\hline & Patients & 568 & 10 & $(0.02)$ & 148 & $(0.26)$ & 410 & $(0.72)$ & & 168 & $(0.15)$ & 968 & $(0.85)$ & \\
\hline & Controls & 561 & 18 & $(0.03)$ & 129 & $(0.23)$ & 414 & $(0.74)$ & 0.95 & 165 & $(0.15)$ & 957 & $(0.85)$ & 0.96 \\
\hline \multicolumn{3}{|c|}{ IVS1-67A/G (rs3749449) } & \multicolumn{2}{|c|}{ GG } & \multicolumn{2}{|c|}{ AG } & & $\mathrm{A}$ & & & 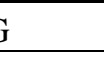 & & $\mathrm{A}$ & \\
\hline & Patients & 570 & 8 & $(0.01)$ & 95 & $(0.17)$ & 467 & $(0.82)$ & & 111 & $(0.10)$ & 1029 & $(0.90)$ & \\
\hline & Controls & 567 & 7 & $(0.01)$ & 89 & $(0.16)$ & 471 & $(0.83)$ & 0.60 & 103 & $(0.09)$ & 1031 & $(0.91)$ & 0.59 \\
\hline IVS1-23G/A (rs & 749448) & & & $\mathrm{AA}$ & & $\mathrm{A}$ & $\mathrm{C}$ & $\mathrm{G}$ & & & A & & $\mathrm{G}$ & \\
\hline & Patients & 569 & 26 & $(0.05)$ & 181 & $(0.32)$ & 362 & $(0.64)$ & & 233 & $(0.20)$ & 905 & $(0.80)$ & \\
\hline & Controls & 566 & 30 & $(0.05)$ & 178 & $(0.31)$ & 358 & $(0.63)$ & 0.75 & 238 & $(0.21)$ & 894 & $(0.79)$ & 0.75 \\
\hline IVS4-35C/T (rs) & 2774) & & & $\mathrm{CC}$ & & $\mathrm{TT}$ & $\mathrm{T}$ & $\mathrm{TT}$ & & &  & & $\mathrm{T}$ & \\
\hline & Patients & 571 & 121 & $(0.21)$ & 260 & $(0.46)$ & 190 & $(0.33)$ & & 502 & $(0.44)$ & 640 & (0.56) & \\
\hline & Controls & 559 & 117 & $(0.21)$ & 268 & $(0.48)$ & 174 & $(0.31)$ & 0.66 & 502 & $(0.45)$ & 616 & $(0.55)$ & 0.65 \\
\hline 1447T/A (rs222 & 902, Phe4331 & & & $\mathrm{AA}$ & & $\mathrm{TT}$ & & $\mathrm{TT}$ & & & is & & $\mathrm{T}$ & \\
\hline & Patients & 575 & 488 & $(0.85)$ & 81 & $(0.14)$ & & $(0.01)$ & & 1057 & $(0.92)$ & 93 & $(0.08)$ & \\
\hline & Controls & 569 & 484 & $(0.85)$ & 82 & $(0.14)$ & 3 & $(0.01)$ & 0.76 & 1050 & $(0.92)$ & 88 & $(0.08)$ & 0.75 \\
\hline 2345C/T (rs148 & 175) & & & $C$ & & $\mathrm{TT}$ & & $\mathrm{TT}$ & & &  & & $\mathrm{T}$ & \\
\hline & Patients & 569 & 120 & $(0.21)$ & 275 & $(0.48)$ & 174 & $(0.31)$ & & 515 & $(0.45)$ & 623 & $(0.55)$ & \\
\hline & Controls & 562 & 118 & $(0.21)$ & 282 & $(0.50)$ & 162 & $(0.29)$ & 0.69 & 518 & $(0.46)$ & 606 & $(0.54)$ & 0.69 \\
\hline 2384A/G (rs148 & 174) & & & $\mathrm{A}$ & & $\mathrm{A}$ & & $\mathrm{G}$ & & &  & & $\mathrm{G}$ & \\
\hline & Patients & 569 & & $(0.03)$ & 150 & $(0.26)$ & 401 & $(0.70)$ & & 186 & $(0.16)$ & 952 & $(0.84)$ & \\
\hline & Controls & 561 & 24 & $(0.04)$ & 162 & $(0.29)$ & 375 & $(0.67)$ & 0.15 & 210 & $(0.19)$ & 912 & $(0.81)$ & 0.14 \\
\hline IVS8-123T/C (r & $62802)$ & & & CC & & C & T & $\mathrm{TT}$ & & &  & & $\mathrm{T}$ & \\
\hline & Patients & 569 & 3 & $(0.01)$ & 91 & $(0.16)$ & 475 & $(0.83)$ & & 97 & (0.09) & 1041 & $(0.91)$ & \\
\hline & Controls & 566 & & $(0.02)$ & 88 & $(0.16)$ & 469 & $(0.83)$ & 0.49 & 106 & $(0.09)$ & 1026 & $(0.91)$ & 0.48 \\
\hline IVS9+15G/A (rs & 280739) & & & $\mathrm{AA}$ & & $\mathrm{A}$ & G & G & & & A & & G & \\
\hline & Patients & 570 & 4 & $(0.01)$ & 84 & $(0.15)$ & 482 & $(0.85)$ & & 92 & $(0.08)$ & 1048 & $(0.92)$ & \\
\hline & Controls & 564 & & $(0.01)$ & 86 & $(0.15)$ & 475 & $(0.84)$ & 0.94 & 92 & $(0.08)$ & 1036 & $(0.92)$ & 0.94 \\
\hline IVS9e & & & & $\mathrm{CC}$ & & $\mathrm{TT}$ & $\mathrm{T}$ & $\mathrm{T}$ & & &  & & $\mathrm{T}$ & \\
\hline & Patients & 96 & 75 & $(0.78)$ & 20 & $(0.21)$ & 1 & $(0.01)$ & & 170 & (0.89) & 22 & $(0.11)$ & \\
\hline & Controls & 95 & & $(0.82)$ & 16 & $(0.17)$ & 1 & $(0.01)$ & 0.53 & 172 & $(0.91)$ & 18 & (0.09) & 0.62 \\
\hline$\overline{\text { IVS9e+1 }}$ & s162777) & & & $\mathrm{CC}$ & & $\mathrm{TT}$ & $\mathrm{T}$ & $T \mathrm{~T}$ & & &  & & $\mathrm{T}$ & \\
\hline & Patients & 570 & 22 & $(0.04)$ & 186 & $(0.33)$ & 362 & $(0.64)$ & & 230 & $(0.20)$ & 910 & $(0.80)$ & \\
\hline & Controls & 566 & & $(0.04)$ & 178 & $(0.31)$ & 365 & $(0.64)$ & 0.82 & 224 & $(0.20)$ & 908 & $(0.80)$ & 0.82 \\
\hline $2912 \mathrm{~T} / \mathrm{C}$ & & & & {$[\mathrm{T}$} & & $\mathrm{C}$ & & $\mathrm{CC}$ & & & $F$ & & $\mathrm{C}$ & \\
\hline & Patients & 96 & 78 & $(0.81)$ & 18 & $(0.19)$ & 0 & $(0.00)$ & & 174 & $(0.91)$ & 18 & (0.09) & \\
\hline & Controls & 95 & & $(0.87)$ & 12 & (0.13) & 0 & $(0.00)$ & 0.32 & 178 & (0.94) & 12 & $(0.06)$ & 0.34 \\
\hline 3175C/T (rs98 & 779) & & & $\mathrm{CC}$ & & $\mathrm{TT}$ & & $\mathrm{T}$ & & & $E$ & & $\mathrm{~T}$ & \\
\hline & Patients & 574 & 25 & $(0.04)$ & 203 & $(0.35)$ & 346 & $(0.60)$ & & 253 & $(0.22)$ & 895 & $(0.78)$ & \\
\hline
\end{tabular}




\begin{tabular}{|c|c|c|c|c|c|c|c|c|c|c|c|c|c|}
\hline Controls & 567 & 23 & $(0.04)$ & 189 & $(0.33)$ & 355 & $(0.63)$ & 0.44 & 235 & $(0.21)$ & 899 & $(0.79)$ & 0.44 \\
\hline 3292(A)3-4 (rs3840229) & & \multicolumn{2}{|c|}{33} & \multicolumn{2}{|c|}{34} & \multicolumn{2}{|c|}{44} & & \multicolumn{2}{|c|}{3} & \multicolumn{2}{|r|}{4} & \\
\hline Patients & 96 & 80 & $(0.83)$ & 16 & $(0.17)$ & 0 & $(0.00)$ & & 176 & $(0.92)$ & 16 & $(0.08)$ & \\
\hline
\end{tabular}

Genotypic $p$ was calculated by Armitage's Trend Test and allelic $p$ was calculated by Fisher's exact test. 
Table 2. Association of the 371T/C polymorphism (rs3749380) in the GRM7 gene with schizophrenia



Genotypic $p$ was calculated by Armitage’s Trend Test and allelic $p$ was calculated by Fisher's exact test.

*Odd ratio $=1.12,95 \%$ confidence interval $=1.03-1.22$ 
A)

$-1889(\mathrm{CAA})_{4-5}$

$-1006 \mathrm{G} / \mathrm{C}_{(\mathrm{rs} 17046184)}$

$-445 \mathrm{C}_{7-8} \quad$ IVS1+781(TAACTGC) I/D $\mathrm{D}_{(\mathrm{rs} 34216931)}$

W



1938C/T 2384G/A

\begin{tabular}{|l|l|}
$N_{(\mathbf{r s} 3749380)}$ & IVS1-67A/G
\end{tabular}

$\downarrow \quad$ IVS1-23G/A $($ (rs 3749448$)$
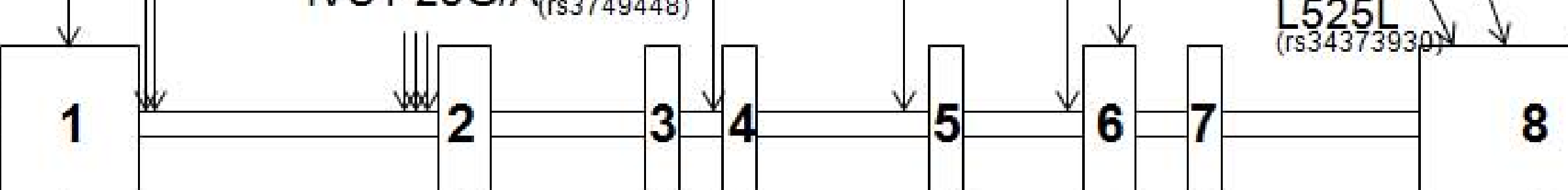

$\uparrow$ ATG

\section{CpG Island}


IVS9e+97C/T

IVS9e+131 C/T

B)

C)



1 28
57

24



2
53

$\times 43$
5

85
6

6

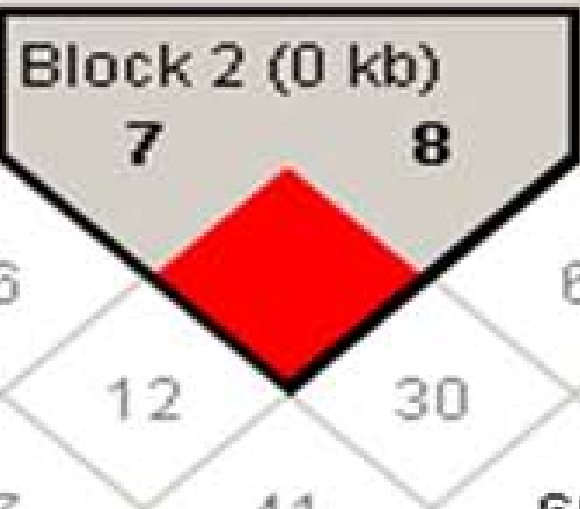

9

71
IVS8+114T/C (rs1485172)

$3175 \mathrm{C} / \mathrm{T}$

3175C/T (rs9826579)


10 $880 \mathrm{~Kb}$

\section{.}






Figure 2 